AperTO - Archivio Istituzionale Open Access dell'Università di Torino

\title{
Geographical variability in survival of European children with central nervous system tumours
}

\section{This is the author's manuscript}

Original Citation:

\section{Availability:}

This version is available http://hdl.handle.net/2318/1681595

since 2020-05-08T11:18:53Z

Published version:

DOI:10.1016/j.ejca.2017.05.028

Terms of use:

Open Access

Anyone can freely access the full text of works made available as "Open Access". Works made available under a Creative Commons license can be used according to the terms and conditions of said license. Use of all other works requires consent of the right holder (author or publisher) if not exempted from copyright protection by the applicable law. 


\section{Geographical variability in survival of European children with central nervous system tumours.}

Gatta $\mathrm{G}^{1}$, Peris-Bonet $\mathrm{R}^{2}$, Visser $\mathrm{O}^{3}$, Stiller $\mathrm{C}^{4}$, Marcos-Gragera $\mathrm{R}^{5}$, Sánchez $\mathrm{MJ}^{6}$, Lacour B ${ }^{7}$, Kaatsch $\mathrm{P}^{8}$, Berrino $\mathrm{F}^{9}$, Rutkowski $\mathrm{S}^{10}$, Botta $\mathrm{L}^{11}$; EUROCARE-5 Working Group. (M.HacklN.ZielonkeW.OberaignerE.Van

EyckenK.HenauZ.ValerianovaN.DimitrovaM.SekerijaH.Storm ${ }^{\mathrm{m}}$ G.Engholm ${ }^{\mathrm{m}}$ M.Mägi ${ }^{\mathrm{n}}$ T.Aareleid ${ }^{\mathrm{n}}$ N.MalilaK.SeppäJ.Faivre ${ }^{1}$ N.Bossard ${ }^{\mathrm{ol}}$ Z.Uhry ${ }^{\mathrm{p}} \mathrm{M}$.Col onna ${ }^{1}$ J.ClavelB.LacourE.DesandesH.Brenner ${ }^{\mathrm{q}}$ P.KaatschA.Katalinic ${ }^{1}$ M.GaramiZ.JakabH.Comber ${ }^{\mathrm{r}}$ G.MazzoleniA.BulatkoC.Buzzoni ${ }^{\mathrm{s}}$ A.GiacominA.Sut era

SardoP.MancusoS.FerrettiA.BarchielliA.CaldarellaG.Gatta ${ }^{\mathrm{tl}}$ M.Sant ${ }^{\mathrm{tl}}$ H.Amash ${ }^{\mathrm{t}}$ C.Amati ${ }^{\mathrm{t}}$ P.Baili ${ }^{\mathrm{tl}}$ F. Berrino ${ }^{\mathrm{tl}}$ S.Bonfarnuzzo ${ }^{\mathrm{t}}$ L. Botta ${ }^{\mathrm{t} R}$.Capocaccia ${ }^{\mathrm{tl}} \mathrm{F}$.Di Salvo R.Foschi' ${ }^{t}$.MarguttitE.Meneghinit P.Minicozzi ${ }^{t}$ A.Trama ${ }^{t}$ D.Serraino ${ }^{\mathrm{u}}$ A.Zucchetto ${ }^{\mathrm{u}}$ R.De

Angelis ${ }^{\mathrm{vl}}$ M.Caldora ${ }^{\mathrm{v}}$ E.Carrani ${ }^{\mathrm{v}}$ S.Francisci ${ }^{\mathrm{v} 1}$ S.Mallone ${ }^{\mathrm{v}}$ D.Pierannunzio ${ }^{\mathrm{v}}$ P.Roazzi ${ }^{\mathrm{v}}$ S.Rossi ${ }^{\mathrm{v}}$ M.Santaquilani ${ }^{\mathrm{v}}$ A.Tavilla ${ }^{\mathrm{v}}$ F.PannozzoS.BuscoR.A.FilibertiE .MaraniP.RicciC.PascucciM.AutelitanoG.SpagnoliC.CirilliM.FuscoM.F.VitaleM.UsalaF.VitaleB.RavazzoloM.MichiaraF.MerlettiM.MauleR.Tumino L.MangoneE.Di

FeliceF.FalciniA.IannelliO.SechiR.CesaraccioS.Piffer ${ }^{\mathrm{w}}$ A.MadedduF.TisanoS.MasperoA.C.FanettiP.CandelaT.ScuderiF.StracciF.BianconiG.Tagliabu $e^{t}$ P.Contiero ${ }^{t}$ M.RuggeS.GuzzinatiS.PildavaG.SmailyteN.Calleja ${ }^{x}$ D.Agius ${ }^{x}$ T.B.JohannesenJ.RachtanS.GóźzźR.MężykJ.BłaszczykM.BębenekM.Bielsk a-Lasota ${ }^{\mathrm{y} 1}$ G.Forjaz de LacerdaM.J.BentoC.CastroA.MirandaA.Mayer-da-SilvaC.Safaei DibaM.Primic-Zakelj ${ }^{21}$ M.ErrezolaJ.BidaurrazagaM.Vicente RanedaJ.M.Díaz GarcíaA.I.Marcos-NavarroR.Marcos-Gragera ${ }^{1}$ A.Izquierdo FontM.J.SanchezD.Y.L.ChangC.NavarroM.D.ChirlaqueC.MorenoIribasE.ArdanazR.Peris-Bonet ${ }^{\text {aa } E . P a r d o ~}$

Romaguera ${ }^{\text {aa } J . G a l c e r a n M . C a r u l l a M . L a m b e ~}{ }^{\text {abl } 1}$ M.MousaviC.BouchardyM.UselS.M.EssH.FrickM.LorezC.HerrmannA.BordoniA.SpitaleI.Konzelmann O.VisserM.AartsR.Otter ${ }^{1}$ M.Coleman ${ }^{\text {acl }}$ C.Allemani ${ }^{\text {ac }}$ B.Rachet ${ }^{\text {ac }}$ J.Verne ${ }^{1}$ C.Stiller ${ }^{\text {ad }}$ A.GavinC.DonnellyD.H.Brewster)

${ }^{\mathrm{m}}$ Danish Cancer Society, Denmark

${ }^{\mathrm{n}}$ National Institute for Health Development, Estonia

${ }^{\circ}$ Hospices Civils de Lyon, France

${ }^{\mathrm{p}}$ Hospices Civils de Lyon, Institut de Veille Sanitaire, France

${ }^{\mathrm{q}}$ German Cancer Research Center, Germany

'National Cancer Registry Ireland, Ireland

${ }^{\mathrm{s}}$ Associazione Italiana Registri Tumoure, Italy

tFondazione IRCCS Istituto Nazionale dei Tumouri, Milan, Italy

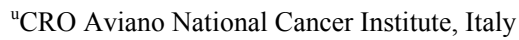

"Istituto Superiore di Sanità, Rome, Italy

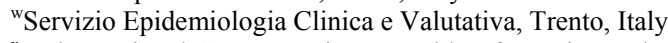

${ }^{\mathrm{x}}$ Malta National Cancer Registry, Health Information and Research, Malta

${ }^{y}$ National Institute of Public Health-NIH, Warszawa, Poland

${ }^{\mathrm{z}}$ Cancer Registry of Republic of Slovenia, Slovenia

${ }^{a}$ aa Spanish Registry of Childhood Tumours, RETI-SEHOP, Spain

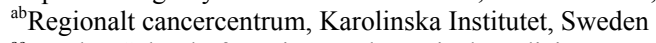

${ }^{\text {ac }}$ London School of Hygiene and Tropical Medicine, UK

${ }^{a d}$ Childhood Cancer Research Group, Oxford, UK

${ }^{a}$ Evaluative Epidemiology Unit, Fondazione IRCCS Istituto Nazionale dei Tumori, Milan, Italy

${ }^{\mathrm{b}}$ Spanish Registry of Childhood Tumours (RETI-SEHOP), Universidad de Valencia, Valencia, Spain

${ }^{\mathrm{c}}$ Netherlands Comprehensive Cancer Organization, Utrecht, The Netherlands

${ }^{\mathrm{d} N a t i o n a l ~ C a n c e r ~ R e g i s t r a t i o n ~ a n d ~ A n a l y s i s ~ S e r v i c e, ~ P u b l i c ~ H e a l t h ~ E n g l a n d, ~ O x f o r d, ~ U K ~}$

${ }^{\circ}$ Epidemiology Unit and Girona Cancer Registry, Oncology Coordination Plan, Department of Health, Government of Catalonia, Catalan Institute of Oncology, Girona, Spain

${ }^{\mathrm{f}}$ Escuela Andalusa de Salud Pública, Instituto de Investigación Biosanitaria ibs, Granada, Hospitales Universitarios de Granada/Universidad de Granada, Granada, Spain

${ }^{\mathrm{g}}$ CIBER de Epidemiología y Salud Pública (CIBERESP), Madrid, Spain

${ }^{\text {h}}$ French National Registry of Childhood Solid Tumours, CHU, Nancy, France

${ }^{\mathrm{i}}$ INSERM, UMRS-1153, CRESS Team 7, University of Paris-Sorbonne, Paris, France

${ }^{\mathrm{j}}$ German Childhood Cancer Registry, Institute for Medical Biostatistics, Epidemiology and Informatics, University of Mainz, Mainz, Germany

${ }^{\mathrm{k}}$ Department of Preventive and Predictive Medicine, Fondazione IRCCS Istituto Nazionale dei Tumori, Milan, Italy

'Department of Pediatric Haematology and Oncology, University Medical Center Hamburg-Eppendorf, Germany

\section{Abstract}

Survival for childhood central nervous system (CNS) tumours varies across Europe, partly because of the difficulty of distinguishing malignant from non-malignant disease. This study examines bias in CNS tumours survival analysis to obtain the reliable and comparable survival figures. 
We analysed survival data for about 15,000 children (age $<15)$ diagnosed with CNS between 2000 and 2007, from 71 population-based cancer registries in 27 countries. We selected high-quality data based on registry-specific data quality indicators and recorded observed 1-year and 5-year survival by countries and CNS entity.

We provided age-adjusted survival and used a Cox model to calculate the hazard ratios (HRs) of death, adjusting by age, site and grading by country.

Recording of non-malignant lesions, use of appropriate morphology codes and completeness of life status follow-up differed among registries. Five-year survival by countries varied less when nonmalignant tumours were included, with rates between $79.5 \%$ and $42.8 \%$. The HRs of dying, for registries with good data, adjusting by age and grading, were between 0.7 and 1.2; differences were similar when site (supra- and infra-tentorial) was included.

Several sources of bias affect the correct definition of CNS tumours, the completeness of incidence series and the goodness of follow-up. The European Network of Cancer Registries needs to improve childhood cancer registration and stress the need to update the International Classification for Cancer. Since survival differences persisted even when restricting the analysis to registries with satisfactory data, and since diagnosis of CNS tumours is difficult and treatment complex, national plans must aim for the revision of the diagnosis and the coordination of care, with adequate national and international networks.

\section{Introduction}

The central nervous system (CNS) is the most common site of solid tumours affecting children [1]. Five-year survival of children with malignant CNS tumours in Europe in 2005-2007 was 58\%, from 54\% in Eastern regions and the UK and Ireland to $65 \%$ in the North [2]. There is presumably ample room for improvement in regions with low survival. However, data on CNS tumours collected by European population-based cancer registries (CRs) are not completely comparable. In a previous analysis of European childhood cancer survival, the differences in registration criteria were so extensive that CNS tumours had to be removed from the analysis of all childhood cancers combined for reliable comparison of survival across countries [2].

We analysed the main sources of bias in childhood CNS tumour survival across Europe, considering the completeness of incidence series, standardisation of the definition of disease entities, the collection and completeness of benign and borderline lesions, and the quality of followup. The major aim was to produce more reliable survival figures for CNS tumours by country, eliminating as far as possible biases affecting comparisons, to illustrate survival variability between countries.

\section{Materials and methods}

\subsection{Study design and data collection}

The EUROCARE-5 database [2] covers about 38,000 CNS tumours, defined as group III in the International Classification of Childhood Cancers, third edition (ICCC-3) [3], diagnosed in European children aged 0-14 years from 1-Jan-1978 to 31-Dec-2007, with vital status updated to 31-Dec-2008. We obtained data from 71 population-based CRs in 27 countries (Table 1). Most countries had national cancer registration. All registries sent data for anonymous central analysis according to a standardised protocol [4]. 


\begin{tabular}{|c|c|c|c|c|c|c|c|c|c|c|c|c|c|}
\hline Area & Country & $\begin{array}{l}\text { Number } \\
\text { of cases } \\
\text { excluded } \\
\text { for major } \\
\text { errors }^{\mathrm{a}}\end{array}$ & $\begin{array}{c}\text { DCO } \\
(\%)\end{array}$ & $\begin{array}{c}\text { Autopsy } \\
\text { (\%) }\end{array}$ & $\begin{array}{c}\text { Alive } \\
\text { with no } \\
\text { survival } \\
\text { time } \\
(\%)\end{array}$ & $\begin{array}{c}\text { Number } \\
\text { of cases } \\
\text { included } \\
\text { in the } \\
\text { analysis }\end{array}$ & $\begin{array}{l}\text { MV } \\
(\%)\end{array}$ & $\begin{array}{c}\text { Alive } \\
2000-03 \\
\text { censored } \\
\text { before } 5 \\
\text { years } \\
(\%)^{b}\end{array}$ & $\begin{array}{c}\text { Non- } \\
\text { malignant } \\
\text { CNS cases } \\
(\%)\end{array}$ & $\begin{array}{l}\text { Unspecified } \\
\text { NOS (ICCC } \\
\text { IIIf) as a } \\
\text { percentage of } \\
\text { the cases in } \\
\text { the analysis } \\
\text { (overall) }\end{array}$ & $\begin{array}{c}\text { Glioma } \\
\text { NOS } \\
\text { (ICD-O } \\
\text { M } \\
9380 / 3) \\
(\%)^{\mathrm{c}}\end{array}$ & $\begin{array}{l}\text { Number } \\
\text { of cases }\end{array}$ & $\begin{array}{l}\text { al CNS } \\
\text { ours }^{\mathrm{d}} \\
\text { Five- } \\
\text { year } \\
\text { survival } \\
\text { (\%) and } \\
\text { (95\% } \\
\text { CI) }\end{array}$ \\
\hline \multirow{4}{*}{$\begin{array}{l}\text { Northern } \\
\text { Europe }\end{array}$} & Denmark & 0 & 0.0 & 0.0 & 0.0 & 264 & 83.0 & 0.0 & 51.9 & 18.6 & n.a & - & n.a \\
\hline & Finland & 0 & 0.0 & 0.0 & 0.0 & 292 & 97.3 & 0.0 & 23.6 & 3.8 & 45.6 & 5 & 0 \\
\hline & Norway & 0 & 0.0 & 0.0 & 0.0 & 335 & 84.2 & 0.0 & 48.1 & 10.4 & 6.9 & 12 & $\begin{array}{l}16.7 \\
(0.2-41)\end{array}$ \\
\hline & Ireland & 1 & 0.4 & 0.0 & 0.0 & 224 & 79.5 & 0.0 & 37.9 & 4.0 & 10.3 & 8 & $\begin{array}{l}25.0(4- \\
56)\end{array}$ \\
\hline \multirow{5}{*}{$\begin{array}{l}\text { UK and } \\
\text { Ireland }\end{array}$} & $\begin{array}{l}\text { UK- } \\
\text { England/Wales }\end{array}$ & 13 & 0.2 & 0.2 & 0.2 & 2460 & 79.4 & 0.9 & 41.2 & 4.3 & 8.7 & 203 & $\begin{array}{l}14.2 \\
(10-20)\end{array}$ \\
\hline & $\begin{array}{l}\text { UK-Northern } \\
\text { Ireland }\end{array}$ & 0 & 0.0 & 0.0 & 0.0 & 103 & 71.8 & 0.0 & 41.7 & 27.2 & 1.0 & 5 & 0 \\
\hline & UK-Scotland & 1 & 0.0 & 0.4 & 0.0 & 245 & 76.3 & 0.0 & 44.1 & 8.2 & 7.4 & 14 & 0 \\
\hline & Austria & 0 & 0.0 & 0.0 & 0.0 & 349 & 95.7 & 0.0 & 25.2 & 4.0 & 7.7 & 45 & $\begin{array}{l}24.1 \\
(13-38)\end{array}$ \\
\hline & Belgium & 5 & 0.0 & 0.0 & 1.7 & 295 & 94.9 & 0.0 & 34.6 & 2.7 & 3.4 & 27 & $\begin{array}{l}12.2(2- \\
33)\end{array}$ \\
\hline \multirow{6}{*}{$\begin{array}{l}\text { Central } \\
\text { Europe }\end{array}$} & France & 5 & 0.0 & 0.0 & 0.2 & 3156 & 85.6 & 3.7 & 43.6 & 1.5 & 7.3 & 219 & $\begin{array}{l}17.9 \\
(13-24)\end{array}$ \\
\hline & Germany & 39 & 0.0 & 0.0 & 1.2 & 3315 & 87.5 & 27.1 & 40.4 & 1.3 & 6.1 & 398 & $\begin{array}{l}19.4 \\
(15-24)\end{array}$ \\
\hline & Switzerland & 2 & 0.0 & 1.3 & 1.3 & 84 & 89.3 & 10.5 & 41.7 & 10.7 & 1.2 & 6 & 0 \\
\hline & $\begin{array}{l}\text { The } \\
\text { Netherlands }\end{array}$ & 2 & 0.0 & 0.2 & 0.0 & 869 & 86.1 & 0.8 & 42.1 & 4.7 & 7.8 & 84 & $\begin{array}{l}20.7 \\
(13-30)\end{array}$ \\
\hline & Croatia & 2 & 0.8 & 0.0 & 0.0 & 260 & 73.1 & 0.0 & 26.2 & 35.0 & 4.2 & 14 & $\begin{array}{l}40.8 \\
(16-65)\end{array}$ \\
\hline & Italy & 1 & 0.1 & 0.0 & 0.0 & 825 & 77.2 & 1.3 & 37.1 & 17.3 & 5.3 & 53 & $\begin{array}{l}28.6 \\
(17-42)\end{array}$ \\
\hline \multirow{7}{*}{$\begin{array}{l}\text { Southern } \\
\text { Europe }\end{array}$} & Malta & 2 & 9.5 & 0.0 & 0.0 & 19 & 84.2 & 0.0 & 26.3 & 0.0 & 5.3 & 3 & $\begin{array}{l}33.3(1- \\
77)\end{array}$ \\
\hline & Portugal & 1 & 0.0 & 0.0 & 0.3 & 292 & 88.4 & 2.0 & 25.4 & 4.5 & 10.3 & 20 & $\begin{array}{l}10.0(2- \\
27)\end{array}$ \\
\hline & Slovenia & 0 & 0.0 & 0.0 & 0.0 & 64 & 96.9 & 0.0 & 34.4 & 4.7 & 3.1 & 10 & $\begin{array}{l}50.0 \\
(18-75)\end{array}$ \\
\hline & Spain & 3 & 0.0 & 0.0 & 0.5 & 579 & 75.9 & 1.6 & 29.3 & 8.6 & 10.2 & 22 & $\begin{array}{l}32.7 \\
(13-54)\end{array}$ \\
\hline & Bulgaria & 24 & 11.6 & 0.0 & 0.0 & 183 & 82.5 & 0.0 & 9.3 & 17.5 & 1.6 & 15 & $\begin{array}{l}20.0(5- \\
42)\end{array}$ \\
\hline & Estonia & 0 & 0.0 & 0.0 & 0.0 & 61 & 77.1 & 0.0 & 37.7 & 21.3 & 0.0 & 1 & 0 \\
\hline & Hungary & 0 & 0.0 & 0.0 & 0.0 & 481 & 93.8 & 2.5 & 34.3 & 0.4 & 7.7 & 21 & $\begin{array}{l}11.7(2- \\
30)\end{array}$ \\
\hline \multirow[t]{4}{*}{$\begin{array}{l}\text { Eastern } \\
\text { Europe }\end{array}$} & Latvia & 12 & 12.2 & 2.4 & 0.0 & 70 & 71.4 & 0.0 & 1.4 & 28.6 & 2.9 & 8 & $\begin{array}{l}70.9 \\
(25-92)\end{array}$ \\
\hline & Lithuania & 3 & 3.7 & 0.0 & 0.0 & 79 & 89.9 & 30.8 & 7.6 & 12.7 & 2.5 & 7 & 0 \\
\hline & Poland & 4 & 0.0 & 0.0 & 2.6 & 149 & 79.9 & 6.4 & 5.4 & 20.1 & 4.7 & 13 & $\begin{array}{l}26.4(7- \\
52)\end{array}$ \\
\hline & Slovakia & 6 & 1.7 & 0.9 & 0.0 & 228 & 88.6 & 0.0 & 42.1 & 9.6 & 1.8 & 6 & $\begin{array}{l}33.3(5- \\
68)\end{array}$ \\
\hline \multicolumn{2}{|c|}{ European pool } & 126 & 0.3 & 0.1 & 0.4 & 15,281 & 87.2 & 8.4 & 38.5 & 5.6 & 7.5 & 1219 & \\
\hline
\end{tabular}

MV, microscopic verification; DCO, death certificate only; CNS, central nervous system; CI, confidence interval; ICD-O M, International Classification of Diseases for oncology third edition; n.a., not available; ICCC, International Classification of Diseases for Oncology, third edition.

In bold type countries with national coverage; partial coverage for Belgium (56\%), Switzerland (29\%), Italy (36\%), Portugal (70\%), Spain (34\%), Poland (12\%). 
aNot correctable errors after consistency check.

bNumber of patients alive followed for less than five years out of all patients diagnosed in 2000-2003 alive before or at five years

cGlioma NOS (in all sites excluding optic nerve).

dAtypical teratoid/rhabdoid tumour (ICD-O M 9508/3), anaplastic astrocytoma (ICD-O M 9401/3), anaplastic oligodendroglioma (ICD-O M 9451/3)

and glioblastoma (ICD-O M 9440/3-9442/3).

Table 2. CNS childhood tumours diagnosed in 2000-07: incidence rates (IRs) and 1- and 5-year survival with 95\% confidence interval (CI) by country and tumour behaviour.

\section{CNS malignant tumours}

Country

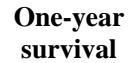

(\%)

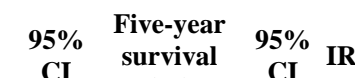

CI

$51-$

survival
(\%)

NS non-malignant tumours

Finland $\quad 86$.

Norway

Irelan

84.5

.3

$\begin{array}{ll}72- & \\ 86\end{array} \quad 60.6$

$\begin{array}{ll}81- & 75.3 \\ 90 & \end{array}$

UK-England 75.8
\& Wales

UK-Northern 78.3

Ireland

UK-Scotland 72.3

Austria $\quad 81$.

Belgium $\quad 76.2$

France $\quad 73$.

Germany $\quad 79.9$

Switzerland 73.5

The

Netherlands

69.2

Croatia

88.0

Italy

Malta

80.8

78.6

Portugal

74.1

Slovenia

78.6

Spain

81.8

Bulgaria

55.4

Estonia

76.3

Hungary

75.5

Latvia

81.2

Lithuania $\quad 77.6$

85.7

Poland

85.7

Slovakia

74.2

European pool 77.1

$\begin{array}{ll}81- & 75.3 \\ 90 & \\ 78- & \\ 89 & 62.5\end{array}$

$$
\begin{array}{lll}
69 & 1.6 & 93.4 \\
69- & & \\
81 & 3 & 97.1
\end{array}
$$$$
\begin{array}{lll}
81 & 3 & 97.1 \\
54 & &
\end{array}
$$$$
\begin{array}{lll}
54- & 2.4 & 96.9
\end{array}
$$$$
\begin{array}{ll}
69- & 59.7 \\
83 &
\end{array}
$$$$
\begin{array}{lll}
50- & 2 & 97.7
\end{array}
$$$$
\begin{array}{lll}
54- & 2.1 \quad 95.3
\end{array}
$$$$
\begin{array}{ll}
74- & 57.1 \\
78 &
\end{array}
$$$$
\begin{array}{ll}
66- & \\
87 & 55.1
\end{array}
$$$$
\begin{array}{ll}
64- & 52.2
\end{array}
$$$$
79
$$$$
\begin{array}{ll}
76- & \\
86
\end{array}
$$$$
86
$$$$
\begin{aligned}
& 70- \\
& 82
\end{aligned}
$$$$
\begin{array}{ll}
71- & \\
75 & 51.8
\end{array}
$$$$
\begin{array}{ll}
78- & \\
82 & 59.1
\end{array}
$$

60

$$
\begin{array}{lll}
60 & 2.1 & 95.3 \\
41- & & \\
67 & 2.1 & 95.4
\end{array}
$$$$
\begin{array}{llll}
67 & & & \\
43- & & & \\
61 & & 97.2
\end{array}
$$$$
\begin{array}{lll}
43- & 1.9 & 97.2 \\
61 & & \\
57- & 2.5 & 100 \\
70 & &
\end{array}
$$

87

$\begin{array}{ll}89- & 94.5 \\ 99 & \end{array}$

$\begin{array}{ll}99 & \\ 93- & 94.7 \\ 99 & \end{array}$

$\begin{array}{ll}91- & 89.3 \\ 99 & \end{array}$

99

94- 927

$96 \quad 92.7$

$\begin{array}{ll}83- & 92.3\end{array}$

$\begin{array}{ll}92- & 92.8 \\ 99 & \end{array}$

$$
94.2
$$$$
\begin{array}{lll}
57- & 2.4 & 96.1
\end{array}
$$$$
\begin{array}{ll}
90- & 93.1 \\
99 &
\end{array}
$$$$
\begin{array}{ll}
99 & 93 . \\
97- &
\end{array}
$$$$
\begin{array}{lll}
49- & 2.1 & 97.9
\end{array}
$$$$
\begin{array}{lll}
57- & & \\
62 & 98
\end{array}
$$$$
\begin{array}{ll}
59- & 57.8 \\
84 &
\end{array}
$$$$
\begin{array}{lll}
42- & 1.7 & 100
\end{array}
$$$$
\begin{array}{ll}
97- & 95.8
\end{array}
$$$$
\begin{aligned}
& 97- \\
& 99
\end{aligned} 96
$$$$
99
$$$$
\begin{array}{lll}
42- & 2.197 \\
51 & &
\end{array}
$$$$
\begin{array}{ll}
65- & 46.4
\end{array}
$$$$
83-\quad 69.6
$$$$
\begin{aligned}
& 92 \\
& 77-
\end{aligned}
$$$$
\begin{array}{ll}
77- & 61.3 \\
84
\end{array}
$$$$
\begin{array}{llll}
9.6 & 62- & 3.2 & 97.1
\end{array}
$$$$
\begin{aligned}
& 84 \\
& 47-
\end{aligned}
$$

61.3

$$
\begin{array}{llll}
57- & & \\
66 & 2.498
\end{array}
$$$$
\begin{array}{lll}
66 & 2.4 & 98 \\
29- & &
\end{array}
$$$$
\begin{aligned}
& 93 \\
& 61 . \\
& 68-
\end{aligned}
$$$$
\begin{array}{ll}
68- & 53.4 \\
79 & \\
63- &
\end{array}
$$

1.2

96

$\begin{array}{lll}46- & 2.6 & 91.9\end{array}$

100

$99 \quad 87.8$

$\begin{array}{ll}70- & 83.7\end{array}$

$\begin{array}{ll}98 & 83.7 \\ 91- & \end{array}$

$91-$
99

$\begin{array}{ll} & 70 \\ 92- & \\ 99 & 97.9\end{array}$

$96-\quad 94.4$

$\begin{array}{llll} & & (\%) & \\ 88- & 92.6 & 87- & 1.7 \\ 97 & 92.6 & 96\end{array}$

$\begin{array}{ll} & 100 \\ 95- & 95.7 \\ 98 & \\ 89- & 93.7 \\ 99 & \\ 96- & 96 \\ 99 & \\ & \\ & \\ & \end{array}$

$\begin{array}{lll}83- & 0.9 & 88.7\end{array}$

$$
\begin{array}{lll}
90- & 2.2 & 90.5
\end{array}
$$

CNS malignant and non-malignant tumours

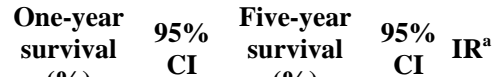

(\%) $\quad$ CI (\%)

$\begin{array}{lll}79- & & \\ 95 & 1.3 & 84.8\end{array}$

$\begin{array}{llll}83- & 77.1 & 71- & 3.3 \\ 91 & & 82 & \end{array}$

$\begin{array}{llll}85- & 79.5 & 74- & 4 \\ 92 & 79.5 & 84 & 4\end{array}$

$\begin{array}{llll}87- & 78.3 & 73- & \\ 93 & 83 & \end{array}$

$\begin{array}{llll}93 & 78.3 & 83 & 4.6\end{array}$

$\begin{array}{llll}79- & 70.9 & 64- & 3.3 \\ 90 & & 77\end{array}$

$\begin{array}{lll}91- & 1.5 & 83.8 \\ 94 & & \end{array}$

$\begin{array}{lll}78- & 1.5 & 85.4 \\ 98 & \end{array}$

$\begin{array}{lll}85- & 1.5 & 83.3\end{array}$

$\begin{array}{lll}97 & & \\ 85- & 0.8 & 86.3\end{array}$

$\begin{array}{llll}82- & 71.8 & 70- & 3.6 \\ 85 & 71.8 & 74 & \end{array}$

$\begin{array}{llll}85 & 71.8 & 74\end{array}$

$\begin{array}{llll}77- & 70.5 & 60- & 3.6 \\ 91 & 79 & \end{array}$

$\begin{array}{llll}78- & 69.8 & 63- & 3.5 \\ 87 & 69.8 & 76 & \end{array}$

$\begin{array}{ll}87 & 76 \\ 82- & 67-\end{array}$

$\begin{array}{llll}82- & 72.1 & 67- & 3.3 \\ 90 & & 77 & \end{array}$

$\begin{array}{llll}78- & & 68- & 3.7 \\ 87 & 74.1 & 79 & 3.7\end{array}$

$\begin{array}{lll}86- & 1.3 & 83.1 \\ 97\end{array}$

$\begin{array}{lll}95- & 1.6 & 83.9\end{array}$

$\begin{array}{lll}95- & 1.4 & 87.2\end{array}$

$\begin{array}{llll}83- & 70.8 & 69- & 3.8 \\ 85 & & 73 & \end{array}$

$86-\quad 73.9 \quad 72-$

$\begin{array}{llll}86- & 73.9 & 72- & 3.4 \\ 88 & & 76 & \end{array}$

$\begin{array}{lll}75- & 75 & 64- \\ 91 & & 2.9\end{array}$

1.284 .4

$\begin{array}{lll}93- & 1.5 & 80.9 \\ 97 & & \\ 84- & 1.1 & 90.4 \\ 98 & & \\ 93- & 1.4 & 87.2 \\ 98 & & \\ & 0.8 & 84.2\end{array}$

$91 \quad 75$

$\begin{array}{llll}78- & 67.0 & 64- & 3.6 \\ 83 & & 70 & \end{array}$

$\begin{array}{llll}86- & 75.6 & 69- & 4.4 \\ 93 & & 81 & \end{array}$

$\begin{array}{llll}85- & & 74.1 & 71- \\ 89 & 7.8\end{array}$

$\begin{array}{lll}89 & 77 & 3.8 \\ 59- & 71.8 & 44-\end{array}$

$\begin{array}{llll}59- & 71.8 & 44- & 3.2 \\ 95 & & 87 & \end{array}$

$\begin{array}{lllll}83- & 84.9 & 74- & 0.9 & 78.7 \\ 96 & 81 & 91 & \end{array}$

$\begin{array}{lllll}90- & 88.5 & 82- & 1.1 & 85.8 \\ 97 & 83 & 93 & \end{array}$

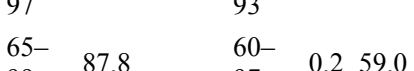

$\begin{array}{lll}97 & 0.2 & 59.0 \\ 56- & & \\ 95 & 1.3 & 82.0\end{array}$

$\begin{array}{lll}95- & 1.3 & 82.0 \\ 86- & 13 & 82.2\end{array}$

$\begin{array}{lll}86- & 1.3 & 82.2\end{array}$

$0 \quad 81.4$

0.179 .0

$\begin{array}{lll}23- & 0.1 & 86.4\end{array}$

$\begin{array}{lll}92- & 1.4 & 84.2\end{array}$

$94-\quad 1.4 \quad 84.7$ $\begin{array}{llll}74- & 61.4 & 55- & 3.5 \\ 83 & & 67 & \end{array}$

$\begin{array}{llll}75- & & 59- & \\ 92 & 72.4 & 82 & 2.7\end{array}$

$\begin{array}{llll}83- & 70.0 & 66- & 3.7\end{array}$

$\begin{array}{ll}88 & 74 \\ 52- & 35-\end{array}$

$\begin{array}{llll}52- & 42.8 & 35- & 2 \\ 66 & & 50 & \end{array}$

$\begin{array}{llll}70- & 69.9 & 57- & 3.5 \\ 90 & 61 & 81 & \end{array}$

$\begin{array}{lll}79- & 67.6 & 63-\end{array}$

$\begin{array}{llll}85 & 67.6 & 63- & 3.7\end{array}$

$\begin{array}{llll}70- & & 53-8 & 53- \\ 89 & 65.4 & 76 & 2.4\end{array}$

$\begin{array}{llll}67- & 53.1 & 41- & 1.8 \\ 87 & 53.1 & 64 & \end{array}$

$\begin{array}{llll}80- & 62.1 & 53- & 2.4 \\ 91 & 62.1 & 70 & \end{array}$

$\begin{array}{llll}79- & 71.9 & 65- & 3.4 \\ 88 & 71.9 & 77 & \end{array}$

$\begin{array}{lll}84-\quad 71.3 & 71-\quad 3.5\end{array}$ 


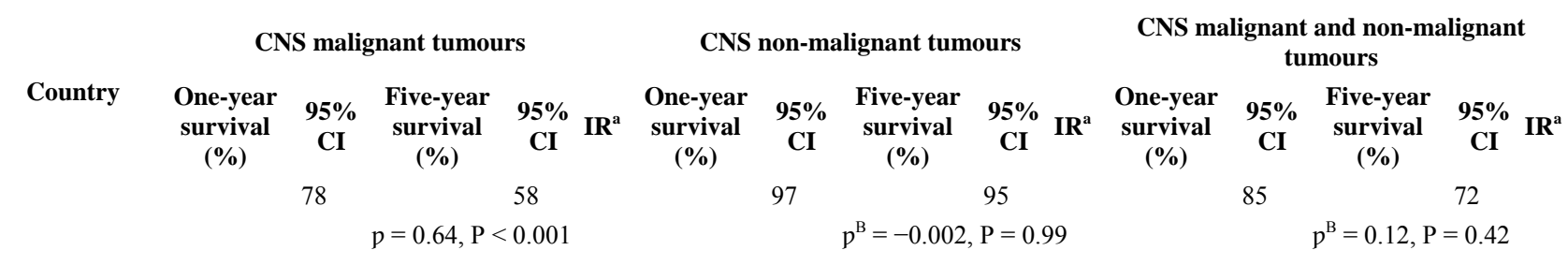

CNS, central nervous system.

$p^{B}$ calculated excluding Austria, Finland, Bulgaria, Latvia, Lithuania and Poland because of incomplete collection of non-malignant cases.

a IR are calculated per 100,000 per year.

Tumours were grouped into the six categories defined by ICCC-3, group III [3], [5]. The EUROCARE-5 protocol [4] asked registries to include both malignant tumours (5th digit in the morphology code equal to 3 in the International Classification of Diseases for Oncology third edition, ICD-O M) and tumours with non-malignant behaviour (5th digit in the morphology codes: 0 or 1). However, some registries communicated to have an incomplete collection of non-malignant tumours (Austria, Bulgaria, Finland, Latvia, Lithuania and Poland).

To analyse survival differences between countries, we had to check the quality of data. For CNS tumour, most important indicators of data quality were: the proportions of unspecified intracranial and intraspinal neoplasms - ICCC-3-IIIf; the proportion of glioma NOS (M-9380/2-3, excluding optical nerve); the proportion of non-malignant tumours, which may suggest, if too low, incomplete registration; the 5-year survival of CNS tumours with very bad prognosis - atypical teratoid/rhabdoid tumours (M-9508/3), anaplastic astrocytoma (M-9401/3), anaplastic oligodendroglioma (M-9451/3) and glioblastoma (M-9440/3-9442/3) which, if higher than average, suggests errors in follow-up.

\subsection{Data analysis}

Observed survival was calculated by the actuarial method. Survival was analysed on a data set containing all childhood cases diagnosed between 1-Jan-2000 and 31-Dec-2007 and followed up until 31-Dec-2008. Survival for 2000-07 was estimated using the complete approach [6]. This is similar to the cohort method but includes recently diagnosed patients (e.g. 2004-2007) with $<5$ years of follow-up. To ensure comparability between countries, age-standardised country-specific 5 -year survivals were also provided. We standardised the estimates to the age distribution of all European children 2000-2007 diagnosed with CNS tumours, defining four classes $(<1,1-4,5-9$ and 10-14 years) [2]. We calculated the crude annual incidence rates (IRs) per 100,000 by country and used Pearson's correlation coefficient $(p)$ to relate these to 5-year survival.

We used a Cox model [7] to calculate the hazard ratios of death (HRs) by country and their 95\% confidence intervals $(95 \% \mathrm{CI})$, according to age, sex, subsite and grading (based on the fourth edition of the WHO classification, which assigned a grade to each ICD-O M, see Table 3) [8]. To adjust for grading, we divided all the tumours as follows: grade I, grade II, grade III-IV, unspecified tumours with non-malignant behaviour, unspecified tumours with malignant behaviour, astrocytoma NOS, glioma NOS (excluding optic nerve). As for the few ICD-O morphological entities not considered in the WHO classification, benign meningioma, pituitary tumour, gliofibroma, choroid glioma, and astroblastoma (even if occasionally it may have an aggressive course), were placed with grade I tumours, and gliomatosi cerebri with grades III-IV. 
Table 3. Five-year survival and 95\% confidence interval (CI) for children with non-malignant or malignant CNS tumours diagnosed in $2000-07$ in Europe by CNS diagnostic group.

\section{Diagnostic group}

IIla ependymoma and choroid plexus tumour

Choroid plexus papilloma

Subependymoma

Myxopapillary ependymoma

Atypical choroid plexus papilloma

Ependymoma, other and NOS

Choroid plexus carcinoma

Anaplastic ependymoma

IIIb astrocytomas

Pilocytic astrocytoma

Subependymal giant cell astrocytoma

Glioma, optic nerve

Pleomorphic xanthoastrocytoma

Fibrillary astrocytoma

Protoplasmic astrocytoma

Gemistocytic astrocytoma

Anaplastic astrocytoma

Glioblastoma and variants

Astrocytomas, NOS

Gliofibroma

IIlc intracranial and intraspinal embryonal

tumour

Medulloblastoma, variants

Medulloblastoma large cell

Desmoplastic/nodular medulloblastoma

PNET, variants $\begin{array}{ccccc}\text { WHO } & \text { Number } & \% & \% & \text { Five-year survival } \\ \text { grade } & & & \text { MV } & (\%)\end{array}$

$95 \% \mathrm{Cl}$

$67-72$

92-99 9390/0

65-99 9383/1

85-99 9394/1

69-97 9390/1

66-74 9391/3, 9393/3

$36-539390 / 3$

56-66 9392/3

79-81

$94-96 \quad 9421 / 1$

97-99 9384/1

$97-99 \quad 9380 / 3$

74-92 9424/3

67-81 9420/3

68-99 9410/3

7-74 9411/3

16-26 9401/3

11-18 9440/3, 9441/3, 9442/3

71-77 9400/3, 9423/3

$9442 / 1$

$3097 \quad 20.799 .2 \quad 57$

$55-59$

62-67 $\begin{aligned} & 9470 / 3,9472 / 3,9480 / 3,9501 / 3, \\ & 9503 / 3\end{aligned}$

$17-56 \quad 9474 / 3$

65-78 9471/3

$36-459473 / 3$ 


\begin{tabular}{|c|c|c|c|c|c|c|c|}
\hline Diagnostic group & $\begin{array}{l}\text { WHO } \\
\text { grade }\end{array}$ & Number & $\%$ & $\begin{array}{c}\% \\
\text { MV }\end{array}$ & $\begin{array}{l}\text { Five-year survival } \\
\text { (\%) }\end{array}$ & $95 \% \mathrm{Cl}$ & 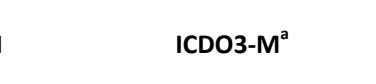 \\
\hline Atypical teratoid/rhabdoid tumour & IV & 258 & 8.3 & 99.2 & 23 & $18-29$ & $9508 / 3$ \\
\hline Illd Other gliomas & & 1642 & 10.9 & 66.1 & 46 & $43-49$ & \\
\hline Oligodendroglioma & II & 212 & 12.9 & 98.1 & 74 & $67-80$ & $9450 / 3,9460 / 3$ \\
\hline Oligodendroglioma, anaplastic & III & 91 & 5.6 & 98.9 & 30 & $20-40$ & $9451 / 3$ \\
\hline Glioma, mixed & III & 137 & 8.4 & 99.3 & 54 & $45-62$ & $9382 / 3$ \\
\hline Astroblastoma & - & 19 & 1.2 & 100 & 78 & $53-92$ & $9430 / 3$ \\
\hline Chordoid glioma & - & 1 & 0.1 & 100 & & & $9444 / 1$ \\
\hline Gliomatosi cerebri & - & 30 & 1.8 & 86.7 & 32 & $16-49$ & $9381 / 3$ \\
\hline Glioma NOS (excl.optic nerve) & - & 1152 & 70.2 & 53 & 41 & $38-44$ & $9380 / 3$ \\
\hline Ille Other specified CNS tumours & & 1866 & 12.4 & 93 & 93 & $91-94$ & \\
\hline Pinealoma and pineocytoma & 1 & 19 & 1.0 & 73.7 & 89 & $43-98$ & $9360 / 1,9361 / 1$ \\
\hline Desmoplastic infantile astrocytoma & 1 & 59 & 3.2 & 98.5 & 85 & $73-92$ & $9412 / 1$ \\
\hline Dysembryoplastic neuroepithelial tumour & 1 & 322 & 17.3 & 88.5 & 99 & $\begin{array}{l}97- \\
100\end{array}$ & $9413 / 0$ \\
\hline Gangliocytomas, ganglioglioma & 1 & 402 & 21.6 & 96.8 & 96 & $93-98$ & $9492 / 1,9505 / 1,9493 / 1$ \\
\hline Meningioma, non-malignant & I & 188 & 10.1 & 92.5 & 95 & $87-96$ & $9530 / 0,9530 / 1,9531 / 0-9539 / 1$ \\
\hline Craniopharyngioma & I & 608 & 32.5 & 93.8 & 97 & $96-99$ & $9350 / 1,9351 / 1,9352 / 1$ \\
\hline Central neurocytoma & II & 17 & 0.9 & 100 & 88 & $61-97$ & $9506 / 1$ \\
\hline Ganglioglioma, anaplastic & III & 25 & 1.3 & 100 & 70 & $48-85$ & $9505 / 3$ \\
\hline Meningioma, malignant & III & 29 & 1.6 & 100 & 79 & $58-90$ & $9530 / 3,9538 / 3,9539 / 3$ \\
\hline Pineoblastoma & IV & 105 & 5.6 & 100 & 46 & $35-56$ & $9362 / 3$ \\
\hline Pituitary tumour & - & 92 & 4.9 & 75 & 100 & & $8270 / 0-8281 / 0,8300 / 0$ \\
\hline IIIf unspecified CNS & & 800 & 5.3 & 17.9 & 64 & $60-67$ & \\
\hline Malignant & - & 429 & 53.4 & 15.2 & 51 & $46-56$ & $8000 / 3-8005 / 3$ \\
\hline Benign & - & 371 & 46.6 & 21 & 78 & $74-82$ & $8000 / 0,1-8005 / 0$ \\
\hline
\end{tabular}

CNS, central nervous system; MV, microscopically verified.

Includes number of cases, proportion of microscopically verified cases and proportion of cancer cases for each diagnostic group. The column headed 'ICDO3-M' lists all the histological ICD0-3 codes in our Data Base different from 0. In this table, we excluded the cases from Denmark.

alnternational Classification of Diseases for Oncology, third edition. 
The conventional two-sided 5\% level was chosen as the threshold for statistical significance. The statistical analyses were performed with STATA [9] and SEER*stat software.

\section{Results}

The data provided, by contributing country, including the basic and specific data quality indicators, are summarised in Table 1. After removing 126 cases known to the registries from death certificate only (DCO) or autopsy, or with no information on follow-up, we finally analysed 15,281 diagnoses of CNS tumours. The proportion of DCO was $0.3 \%$ overall, and less than $1 \%$ in most countries. High DCO rates in Malta, Bulgaria and Latvia were explained by 2, 24 and 10 cases. In all, 87\% of childhood CNS tumours had microscopic verification (MV), ranging from 71 to $72 \%$ in Northern Ireland and Latvia to $97 \%$ in Finland and Slovenia. The proportion of cases censored before five years of follow-up, among 2000-2003 diagnosed cases still alive, was less than 4\% in all countries except Lithuania (31\%), Germany (27\%), Switzerland (10\%) and Poland (6\%). DCO and Autoptic cases did not enter in the survival analyses, whereas censored cases contributed with their period of observation and then exited as 'censored'.

There was $39 \%$ of non-malignant tumours, but with wide differences from $<10 \%$ to $>40 \%$. Table 1 also includes the proportion of CNS tumours with morphologies not specified: the ICCC IIIfunspecified intracranial and intraspinal tumours (UNSP, $6 \%$ of all CNS tumours) and glioma NOS (ICD-O M 9380/3, optic nerve excluded, 8\%). The proportion of UNSP was mostly below 10\%, but high proportions $(>20 \%)$ were registered in Northern Ireland, Latvia, Estonia, Poland and Croatia. The proportions of glioma NOS were mostly $<10 \%$, lower for countries with high UNSP, and higher for those without. Finland, however, had a very high proportion of glioma NOS.

As an indicator of completeness of follow-up across countries, we report 5-year survival for highly lethal tumours (anaplastic astrocytoma, glioblastomas, anaplastic oligodendroglioma and atypical teratoid/rhabdoid tumour) which were $8 \%$ of all CNS tumours. Too high survival figures suggest difficulties in access to death certificates or administrative sources, so some patients are wrongly considered alive only because the death certificates did not reach the registries or did not match the cases, or the patients become untraceable [10]. Slovenia, Latvia and Croatia had high 5-year survival ( $\geq 40 \%$ ), although with wide 95\%CIs. Five-year survival in Europe for this group of tumours was 19\% (not in Table). Unfortunately, Denmark coded CNS tumour morphologies in few generic groups (Neoplasm NOS, Ependymoma NOS, Astrocytoma NOS, Glioma malignant NOS, Medulloblastoma NOS and Ganglioglioma NOS), so we could not estimate some quality indicators in Table 1.

Table 2 shows 1 - and 5 -year survival by country for malignant and non-malignant tumours and for all the tumours combined, with their IRs per 100,000/year. Five-year survival of malignant tumours averaged $57 \%$ in Europe, from $75 \%$ in Finland to $38 \%$ in Bulgaria. Non-malignant tumours had high 5-year survival rates (94\% on average), between $100 \%$ and $85 \%$, except Estonia, Portugal and Poland. For all CNS tumours, survival reached $71 \%$ in Europe and the variability decreased, particularly among non-Eastern European countries. There were no longer any differences in survival within Northern Europe and the UK and Ireland when non-malignant lesions were included. For Central and Southern Europe, differences dropped: 5 -year survival was $\geq 70 \%$ in all except Netherlands (67\%) and Portugal (61\%). For Eastern Europe, Bulgaria, Latvia, Lithuania and Poland, with incomplete registration of non-malignant cases, had low survival $(<66 \%)$, whereas for Estonia, Slovakia and Hungary, which registered non-malignant cases, survival was $70 \%, 72 \%$ and $68 \%$, respectively. Survival in all regions dropped steeply after the first year from diagnosis, so there were large gaps between 1- and 5-year survival. 
The annual IRs (per 100,000/year) of CNS malignant tumours were between 1.6 and 3 . The rates for all CNS tumours combined were highest $(\geq 4)$ in Finland, Croatia and Norway. Rates between 3 and 4 were also reported from all UK and Ireland, and for most of the countries in Southern and Central European countries.

There was a relation between incidence and survival of malignant tumours by country: the higher the incidence, the better the survival $(p=0.6 ; \mathrm{P}<0.001)$. A relation between incidence and survival of all CNS cases was not found, when including only registries that had a complete registration of non-malignant tumours (Table 2).

Five-year survival rates by histotype within the six ICCC groups varied widely both between and within the main groups (Table 3 ). In the category of ependymomas and variant (IIIa), the choroid plexus carcinomas had the lowest survival (44\%); non-malignant tumours, $20 \%$ of all IIIa cases had survival $>89 \%$. Astrocytomas (IIIb) included $14 \%$ of anaplastic astrocytomas and glioblastomas, with poor survival $21 \%$ and $14 \%$. Pilocytic astrocytoma, $53 \%$ of IIIb group, and optic nerve glioma, had both very high outcome (95\% and 99\%). Sixty-five percent of embryonal tumours (ICCC IIIc) were medulloblastoma with $65 \%$ five-year survival. Eighteen percent were other PNET with worse survival (41\%). A small proportion of IIIc were atypical teratoid/rhabdoid tumours, with poor survival $(23 \%)$.

Seventy percent of 'other gliomas' (IIId) were glioma NOS (optic nerve excluded) with 41\% 5-year survival. ICCC IIIe 'other specified CNS tumours' consisted mainly of non-malignant tumours with very good prognosis, except pinealoblastoma. The 'unspecified tumours of the CNS' (IIIf) were half non-malignant and half malignant.

Table 4 shows 5-year survival of grades I and III-IV CNS tumours by country. For grade I, survival was $>90 \%$, except Bulgaria, Portugal and Poland. Grades III-IV had poor survival (49\%), with larger differences across countries, from $<40 \%$ in Bulgaria, Lithuania and Netherlands to $>60 \%$ in Finland, Switzerland, Austria, Croatia and Slovenia. Overall, 5-year survival for grade II was 74\%, $46 \%$ for grade III and $51 \%$ for grade IV (not shown).

Table 4. Five-year survival and 95\% confidence interval (CI) for children with non-malignant or malignant CNS tumours diagnosed in $2000-07$ by country and grade.

\begin{tabular}{|c|c|c|c|c|c|c|}
\hline \multirow{2}{*}{ Country } & \multicolumn{3}{|c|}{ WHO grade I tumours ${ }^{a}$} & \multicolumn{3}{|c|}{ WHO grade III and IV tumours ${ }^{b}$} \\
\hline & Number & r Five-year survival (\%) & $95 \% \mathrm{Cl}$ & Number & Five-year survival (\%) & $95 \% \mathrm{Cl}$ \\
\hline Finland & 60 & 100 & & 49 & 65.6 & $50-78$ \\
\hline Norway & 134 & 94.4 & 89-97 & 96 & 54.2 & $43-64$ \\
\hline Ireland & 96 & 90.3 & $81-95$ & 54 & 52.1 & $37-65$ \\
\hline UK-England/Wales & 1017 & 96 & $94-97$ & 757 & 46 & $42-50$ \\
\hline UK-Northern Ireland & & 96.6 & $78-100$ & 28 & 49.7 & $29-68$ \\
\hline UK-Scotland & 101 & 94.4 & $87-98$ & 75 & 44 & $31-56$ \\
\hline Austria & 91 & 94.4 & $85-98$ & 147 & 60.1 & $51-68$ \\
\hline Belgium & 119 & 94.9 & 89-98 & 106 & 50.8 & $40-60$ \\
\hline France & 1488 & 96.4 & $95-97$ & 1011 & 46.9 & $44-50$ \\
\hline
\end{tabular}




\begin{tabular}{|c|c|c|c|c|c|c|}
\hline \multirow{2}{*}{ Country } & \multicolumn{3}{|c|}{ WHO grade I tumours ${ }^{\mathrm{a}}$} & \multicolumn{3}{|c|}{ WHO grade III and IV tumours ${ }^{b}$} \\
\hline & Number & Five-year survival (\%) & $95 \% \mathrm{Cl}$ & Number & Five-year survival (\%) & ) $95 \% \mathrm{Cl}$ \\
\hline Germany & 1445 & 96.9 & $96-98$ & 1267 & 53.5 & $50-57$ \\
\hline Switzerland & 30 & 100 & & 33 & 60.1 & $41-75$ \\
\hline The Netherland & 359 & 95.9 & $93-98$ & 294 & 39.8 & $38-46$ \\
\hline Croatia & 37 & 94.4 & 79-97 & 75 & 63.2 & $51-73$ \\
\hline Italy & 258 & 97.1 & $94-99$ & 243 & 56 & $49-62$ \\
\hline Malta & 5 & 100 & & 9 & 41.7 & $11-71$ \\
\hline Portugal & 77 & 86.5 & $76-93$ & 106 & 46.5 & $36-56$ \\
\hline Slovenia & 21 & 100 & & 32 & 60.8 & $41-76$ \\
\hline Spain & 161 & 93.8 & $88-97$ & 163 & 50.1 & $41-59$ \\
\hline Bulgaria & 17 & 87.8 & $60-97$ & 76 & 36.2 & $25-48$ \\
\hline Estonia & 13 & 92.3 & $57-99$ & 23 & 51.4 & $30-70$ \\
\hline Hungary & 173 & 92.6 & $87-96$ & 180 & 43.5 & $35-52$ \\
\hline Latvia & 1 & n.e & & 13 & 59.3 & $28-81$ \\
\hline Lithuania & 7 & 100 & & 31 & 37.3 & $20-54$ \\
\hline Poland & 8 & 70 & $23-92$ & 65 & 53.6 & $39-66$ \\
\hline Slovakia & 96 & 97.9 & $92-100$ & 60 & 51.4 & $38-64$ \\
\hline European pool & 5843 & 95.9 & $95-97$ & 4993 & 49.4 & $48-51$ \\
\hline
\end{tabular}

CNS, central nervous system; n.e, not estimable.

a5843 cases, $39 \%$ of all the CNS cases.

b4993 cases, $33 \%$ of all the CNS cases.

After excluding registries stated incomplete collection or with a proportion $\leq 25 \%$ of non-malignant cases, or presented possible classification or follow-up problems, we compared age-adjusted 5-year survival figures for 17 countries (Fig. 1).

Comparing to Table 2, including all the countries, variability was lower but still present, with survival between $78 \%$ (Norway) and 58\% (Portugal).

Table 5 shows the HRs of dying, adjusting by age and grading, both statistically significant in univariate analysis. With England \& Wales as reference, Netherlands had a significantly higher and Norway, Italy and Germany lower risk of dying.

Table 5. Hazard ratio (HR) of dying and 95\% confidence interval (CI) for children with non-malignant or malignant CNS tumours diagnosed in 2000 07 by country, adjusted by age, grading (model 1), and site (supra- and infra-tentorial; model 2). 


\begin{tabular}{|c|c|c|c|c|}
\hline \multirow{3}{*}{ Country } & \multicolumn{2}{|c|}{ Model 1} & \multicolumn{2}{|c|}{ Model 2} \\
\hline & & & & \\
\hline & HR & $95 \% \mathrm{Cl}$ & HR & $95 \% \mathrm{Cl}$ \\
\hline Norway & $0.70^{\mathrm{a}}$ & $0.5-0.9$ & $0.75^{\mathrm{a}}$ & $0.6-0.99$ \\
\hline Belgium & 0.92 & $0.7-1.2$ & 0.95 & $0.7-1.3$ \\
\hline France & 1.10 & $0.99-1.2$ & 1.10 & $0.98-1.2$ \\
\hline Germany & $0.85^{\mathrm{a}}$ & $0.8-0.9$ & $0.88^{\mathrm{a}}$ & $0.8-0.99$ \\
\hline Switzerland ${ }^{b}$ & 0.76 & $0.5-1.2$ & 0.85 & $0.5-1.5$ \\
\hline The Netherlands & $1.20^{\mathrm{a}}$ & $1.03-1.4$ & $1.22^{\mathrm{a}}$ & $1.04-1.4$ \\
\hline Italy $^{b}$ & $0.78^{\mathrm{a}}$ & $0.6-0.9$ & $0.79^{\mathrm{a}}$ & $0.6-0.98$ \\
\hline Malta & 0.87 & $0.4-2.1$ & 1.31 & $0.5-3.5$ \\
\hline Portugal $^{b}$ & 1.13 & $0.9-1.5$ & 1.18 & $0.9-1.6$ \\
\hline Spain $^{b}$ & 0.86 & $0.7-1.02$ & $0.76^{a}$ & $0.6-0.9$ \\
\hline Ireland & 0.99 & $0.8-1.3$ & 0.94 & $0.7-1.3$ \\
\hline England and Wales & REF & & REF & \\
\hline Northern Ireland & 0.88 & $0.6-1.3$ & 0.81 & $0.5-1.3$ \\
\hline Scotland & 1.11 & $0.9-1.4$ & 1.15 & $0.9-1.5$ \\
\hline Slovakia & 0.93 & $0.6-1.5$ & 0.98 & $0.6-1.7$ \\
\hline Estonia & 1.04 & $0.9-1.3$ & 0.99 & $0.8-1.2$ \\
\hline Hungary & 1.01 & $0.8-1.3$ & 0.98 & $0.7-1.3$ \\
\hline
\end{tabular}

CNS, central nervous system; REF, England and Wales was taken as reference group.

aHR statistically significant.

bregistries included: Switzerland: Basel, Geneva, Grisons, St. Gallen, Valais; Italy: Alto Adige, Biella, Catanzaro, Ferrara, Firenze-Prato, Genova, Latina, Mantova, Marche Childhood, Modena, Palermo, Parma, Piemonte Childhood, Ragusa, Romagna, Sassari, Sondrio, Trapani, Veneto; Portugal: Southern Portugal; Spain: Basque country, Cuenca, Girona, Granada, Navarra, Spain Childhood, Valencia Childhood.

Restricting the analysis to supra- and infra-tentorial tumours ( $72 \%$ of all cases) and adjusting the model also for site, divided in two groups, the results remained the same, except Spain with significantly lower risk than England \& Wales. The risks by country did not changed excluding CNS unspecified cases (UNSP, glioma NOS and astrocitoma NOS, 17\% of all cases) from the multivariate analysis (not in table).

\section{Discussion}

This EUROCARE analysis illustrates the difficulties in comparing survival of childhood CNS tumours between countries. First, there was incomplete collection of non-malignant tumours. The 
proportions of non-malignant cases were 44\% in France, $41 \%$ in England \& Wales and 40\% in Germany. We can be confident about the level of completeness of these large childhood CRs, so an acceptable proportion of non-malignant cases should range between about 35\% and 50\%. Among the countries included in Fig. 1 and Table 5, Malta, Portugal and Spain are outliers with possible under registration.

The correct classification of CNS tumours without microscopic confirmation, $13 \%$ in our study, is even more difficult. Nevertheless, the high proportion of microscopically verified CNS tumours does suggest incomplete and selective collection, as we know that part of the lesion can be identified by imaging only. As shown by the national childhood cancer registries, a proportion of microscopically verified cases higher than $90 \%$ should be considered suspicious for selective collection of cases. In our study, five countries were over this threshold, some of them with the highest (Finland) and other with low survival figures (Hungary; Table 2).

Another problem is that the definition of malignancy according to the WHO Classification of CNS tumours may vary between and within countries. It is not easy to distinguish malignant from nonmalignant or low-grade from high-grade tumours. Even in a trial setting with pathological review, $28 \%$ children with glioma were incorrectly diagnosed high grade instead of low grade [11]. Grading is containing a subjective component (Ellison DW et al.[14], Journal of Negative Results Biomed $2011 ; 10: 7)$, actually in entities like ependymoma, there is high variation between grading II and III ependymomas across countries, but no relevant survival difference. Inter-observer variability of the histologic features of anaplasia in CNS tumours illustrates a problem, since histology is so important for diagnostic and therapeutic decisions. Gilles et al.[12] suggested four histologic features as indispensable for brain tumour analysis: necrosis, cell density, nuclear pleomorphism and mitoses. It would be interesting to review a population-based sample of pathological reports of childhood CNS tumours, to see how often these characteristics are included in the report. Again, 'benign' (grade I/II) lesions may behave clinically highly malignant if located in an inoperable CNS location (e.g. brain-stem) and vice-versa (grade IV tumours such as medulloblastoma may have very high survival rates based on its biological subgroup). We adjusted by localisation, but we could not take into account biological subgroup, because this is no contemplate in the used classifications [13], [14]. Therefore, classification such as ICCC should be updated, and this could be realised within the ENCR, possibly in agreement with SIOPE.

Another issue can indicate low quality in disease definition: high proportions of UNSP and the use of unspecific codes like glioma NOS. UNSP and glioma NOS (optic nerve excluded) amounted to $13 \%$ of all CNS tumours, with 5 -year survivals between $64 \%$ and $41 \%$. The high proportion of UNSP suggests low-quality disease definition and the erroneous registration of non-malignant tumours or not biopsied tumours among the CNS cases. Survival figures were therefore presumably overestimates for some countries.

An important issue in survival comparisons is the completeness of follow-up, in terms of capturing all the deaths after diagnosis. In some cases, registries are aware that a patient is no longer traceable or require more time for completing follow-up; this is shown in the proportion of early censored cases, which was highest for Germany (27\%) and Lithuania (31\%). In other cases, no information reaches the registry, so these patients are classified as alive. Dealing with this involves analysing cancers with a very poor prognosis [15]. We studied this by comparing 5-year survival of lethal tumours (Table 1). If the diagnoses were correct, too high survival figures suggest difficulties in access to information from death certificates or administrative sources. Among countries with survival for lethal tumours higher than the European average, Latvia, Slovenia and Croatia had 5year survival of $\geq 40 \%$. Again, a certain overestimation must be considered. 
The data quality problems and differences in registration practice for CNS tumours should draw the attention of the ENCR to the need to improve the standardisation of registry practices and criteria, taking account of modern diagnostic imaging procedures for tumours where MV is not always possible or convenient. We suggest to update the ENCR recommendation on brain tumour [5] by convene a new working group of expert. We are also aware about the difficulties made by the privacy regulations that limited the access to mortality data and in some cases also to the other source of data for cancer registries. Therefore, even if not specific for CNS cancer, the ENCR should continue to stress the solution of this problem.

Even when restricted to the registries considered to have the most fully comparable data, survival analysis of childhood CNS tumours indicated some variability with, compared with England \& Wales, a significant lower risk of dying between $30 \%$ and $15 \%$ (Norway, Germany, Italy). Since Germany had a high percentage of patients not yet followed for five years at the closing date of this study, we performed an analysis excluding all 2000-2003 diagnosed cases not followed for 5 years; this gave similar results, though no longer significant for Germany (not shown). The high proportion of censured cases in Germany may be partly related to the apparent good survival. Similar survival differences were reported in adult CNS tumours for Europe, same diagnosis period. However, variation between countries was lower than those reported for children, but with the Nordic and some central countries with the highest outcome [16].

The complexity of treating childhood CNS tumours is partly responsible for the observed great variability in histological classification, follow-up and registration practices. To improve this situation, requires much greater collaboration between the treating centres and population-based cancer registries and emphasises the need for quality control of pathological diagnoses for both treatment and registration purposes [17]. This might be best achieved through centralisation of diagnosis and treatment in fewer centres, linked through national networks to permit the continuation of therapies and clinical follow-up close to the child's home.

International networks are also vital, especially since continuing progress in biological stratification of these rare tumours can support risk-adapted therapeutic stratification that will improve outcomes and reduce treatment-related morbidity. Since these cancers require a high level of specialisation and sophisticated infrastructure, close collaboration should be fostered between the Eastern countries and the European regions with better survival for childhood CNS tumours, also taking the advantage of the European Commission's call for twinning programs [18]. The implementation and extension of the European directive on Cross-Border Healthcare [19] is also important for European countries with small populations. Some of these have low-income levels, compared with the rest of Europe, and are unlikely to develop the necessary infrastructure within their borders.

\section{Conflict of interest statement}

None declared.

\section{Acknowledgements}

The authors thank J.D. Baggott for editing, R. Capocaccia for his important suggestions and L. Buratti for technical assistance.

\section{Funding}

This work was supported by: 
-Italian Ministry of Health, Research Project title 'Population-based comparisons of cancer burden indicators' [RF-2009-1529710].

-European Commission through the Executive Agency for Health and Consumers [Joint Action No. 20102202]. Project title 'European Partnership for Action Against Cancer' -EPAAC.

-European Commission through the Executive Agency for Health and Consumers [grant no. 2000111201]. Project title 'Information network on rare cancers' - RARECARENet.

-Compagnia di San Paolo Foundation.

\section{Role of funding source}

The funders had no role in study design, collection, analysis or interpretation of data, or writing of the report. The corresponding author had full access to all the data and had final responsibility for the decision to submit for publication.

\section{Appendix.}

\section{EUROCARE-5 Working Group:}

Austria: M. Hackl, N. Zielonke (Austrian CR); W. Oberaigner (Tyrol CR); Belgium: E. Van Eycken, K. Henau (Belgian CR); Bulgaria: Z. Valerianova, N. Dimitrova (Bulgarian CR); Croatia: M. Sekerija (Croatian CR); Denmark: H. Storm, G. Engholm (Danish Cancer Society); Estonia: M. Mägi (Estonian CR; National Institute for Health Development); T. Aareleid (National Institute for Health Development); Finland: N. Malila, K. Seppä (Finnish CR); France: J. Faivre* (Côte d'Or Digestive Tract Registry, Burgundy); N. Bossard* (Hospices Civils de Lyon); Z. Uhry (Hospices Civils de Lyon; Institut de Veille Sanitaire); M. Colonna* (Isère CR); J. Clavel (National Registry of Childhood Hematological Malignancies); B. Lacour, E. Desandes (National Registry of Childhood Solid Tumours); Germany: H. Brenner (German Cancer Research Center); P. Kaatsch (German Childhood CR, Mainz); A. Katalinic* (Schleswig-Holstein CR); Hungary: M. Garami, Z. Jakab (Hungarian Child CR); Ireland: H. Comber (National Cancer Registry Ireland); Italy: G. Mazzoleni, A. Bulatko (Alto Adige CR); C. Buzzoni (Associazione Italiana Registri Tumoure); A. Giacomin (Biella CR); A. Sutera Sardo, P. Mancuso (Catanzaro CR); S. Ferretti (Ferrara CR); A. Barchielli, A. Caldarella (Firenze-Prato CR); G. Gatta*, M. Sant*, H. Amash, C. Amati, P. Baili*, F. Berrino*, S. Bonfarnuzzo, L. Botta, R. Capocaccia*, F. Di Salvo, R. Foschi, C. Margutti, E. Meneghini, P. Minicozzi, A. Trama (Fondazione IRCCS Istituto Nazionale dei Tumouri, Milan); D. Serraino, A. Zucchetto (Friuli Venezia Giulia CR, CRO Aviano National Cancer Institute); R. De Angelis*, M. Caldora, E. Carrani, S. Francisci*, S. Mallone, D. Pierannunzio, P. Roazzi, S. Rossi, M. Santaquilani, A. Tavilla (Istituto Superiore di Sanità, Rome); F. Pannozzo, S. Busco (Latina CR); R. A. Filiberti, E. Marani (Liguria CR, IRCCS AOU SM-IST); P. Ricci (Mantova CR); C. Pascucci (Marche Childhood CR); M. Autelitano (Milano CR); G. Spagnoli, C. Cirilli (Modena $C R$ ); M. Fusco, M. F. Vitale (Napoli 3 South CR); M. Usala (Nuoro CR); F. Vitale, B. Ravazzolo (Palermo CR); M. Michiara (Parma CR); F. Merletti, M. Maule (Piemonte Childhood CR); R. Tumino (Ragusa CR); L. Mangone, E. Di Felice (Reggio Emilia CR); F. Falcini (Romagna CR); A. Iannelli (Salerno CR); O. Sechi, R. Cesaraccio (Sassari CR); S. Piffer (Servizio Epidemiologia Clinica e Valutativa, Trento); A. Madeddu, F. Tisano (Siracusa CR); S. Maspero, A. C. Fanetti (Sondrio CR); P. Candela, T. Scuderi (Trapani CR); F. Stracci, F. Bianconi (Umbria CR); G. Tagliabue, P. Contiero (Varese Province CR, Fondazione IRCCS Istituto Nazionale dei Tumouri); M. Rugge, S. Guzzinati (Veneto CR); Latvia: S. Pildava (Latvian CR); Lithuania: G. Smailyte (Lithuanian CR); Malta: N. Calleja, D. Agius (Malta National Cancer Registry, Health Information 
and Research); Norway: T. B. Johannesen (Norwegian CR); Poland: J. Rachtan (Cracow CR); S. Góźdź, R. Mężyk (Kielce CR); J. Błaszczyk, M. Bębenek (Lower Silesia CR, Wroclaw); M. Bielska-Lasota* (National Institute of Public Health-NIH, Warszawa); Portugal: G. Forjaz de Lacerda (Açores CR); M. J. Bento, C. Castro (Northern Portugal CR); A. Miranda, A. Mayer-daSilva (Southern Portugal CR); Slovakia: C. Safaei Diba (Slovakian National CR); Slovenia: M. Primic-Zakelj* (Cancer Registry of Republic of Slovenia); Spain: N. Larrañaga, A. Lopez de Munain (Basque Country CR); M. Vicente Raneda (Comunitat Valenciana Childhood CR); J. M. Díaz García, A. I. Marcos-Navarro (Cuenca CR); R. Marcos-Gragera*, A. Izquierdo Font (Girona CR); M. J. Sanchez, D. Y. L. Chang (Granada CR, CIBERESP, ibs.Granada); C. Navarro, M. D. Chirlaque (Murcia CR, CIBERESP, IMIB-Arrixaca); C. Moreno-Iribas, E. Ardanaz (Navarra CR, CIBERESP); R. Peris-Bonet, E. Pardo Romaguera (Spanish Registry of Childhood Tumours, RETISEHOP); J. Galceran, M. Carulla (Tarragona CR); Sweden: M. Lambe* (Regionalt cancercentrum and Karolinska Institutet); Switzerland: M. Mousavi (Basel CR); C. Bouchardy, M. Usel (Geneva CR); S. M. Ess, H. Frick (Grisons-Glarus CR); M. Lorez (NICER); S. M. Ess, C. Herrmann (St. Gallen CR); A. Bordoni, A. Spitale (Ticino CR); I. Konzelmann (Valais CR); The Netherlands: O. Visser, M. Aarts (The Netherlands CR); R. Otter*; UK-England: M. Coleman*, C. Allemani, B. Rachet (London School of Hygiene and Tropical Medicine); J. Verne*; UK-England and Wales: C. Stiller (Childhood Cancer Research Group, Oxford); UK-Northern Ireland: A. Gavin, C. Donnelly (Northern Ireland CR); UK-Scotland: D. H. Brewster (Scottish CR).

\section{References}

1. R. Peris-Bonet, C. Martínez-García, B. Lacour, S. Petrovich, B. Giner-Ripoll, A. Navajas, et al.Childhood central nervous system tumors incidence and survival in Europe (1978-1997): report from Automated Childhood Cancer Information System project Eur J Cancer, 42 (13) (2006), pp. 2064-2080

2. G. Gatta, L. Botta, S. Rossi, T. Aareleid, M. Bielska-Lasota, J. Clavel, et al.Childhood cancer survival in Europe 1999-2007: results of EUROCARE-5-a population-based study Lancet Oncol, 15 (1) (2014), pp. 35-47

3. E. Steliarova-Foucher, C. Stiller, B. Lacour, P. KaatschInternational classification of childhood cancer, third edn Cancer, 103 (7) (2005), pp. 1457-1467

4. EUROCARE [Internet]. EUROCARE-5 Protocol for updating population-based cancer survival in Europe [cited 10 November 2016]. Available from: http://www.eurocare.it/LinkClick.aspx?fileticket=JWJdXS_zxZE\%3d\&tabid=89 [page 5].

5. ENCR [Internet].Recommendations for coding Tumours of the Brain and Central Nervous System; distribute in 1998 [cited 2016 November 10]. Available from: http://www.encr.eu/images/docs/recommendations/braincns.pdf.

6. H. Brenner, T. HakulinenUp-to-date cancer survival: period analysis and beyond Int J Cancer, 124 (6) (2009), pp. 1384-1390

7. D.R. CoxRegression models and life tables J R Stat Soc B, 34 (2) (1972), pp. 187-200

8. D.N. Louis, H. Ohgaki, O.D. Wiestler, W.K. Cavenee, P.C. Burger, A. Jouvet, et al.The 2007 WHO classification of tumours of the central nervous system Acta Neuropathol, 114 (2) (2007), pp. 97109

9. StataCorpStata statistical software: release 13 StataCorp LP, College Station, TX (2013)

10. R. De Angelis, M. Sant, M.P. Coleman, S. Francisci, P. Baili, D. Pierannunzio, et al.Cancer survival in Europe 1999-2007 by country and age: results of EUROCARE-5-a population-based study Lancet Oncol, 15 (2014), pp. 23-34

11. M. Fouladi, D.L. Hunt, I.F. Pollack, G. Dueckers, P.C. Burger, L.E. Becker, et al.Outcome of children with centrally reviewed low-grade glioma treated with chemotherapy with or without 
radiotherapy on Children's Cancer Group High-Grade Glioma Study CCG-945 Cancer, 98 (6) (2003), pp. 1243-1252

12. F.H. Gilles, C.J. Tavaré, L.E. Becker, P.C. Burger, A.J. Yates, I.F. Pollack, et al.Pathologist interobserver variability of histologic features in childhood brain tumours: results from the CCG945 study Pediatr Dev Pathol, 11 (2) (2008), pp. 108-117

13. A Gajjar, D.C. Bowers, M.A. Karajannis, S. Leary, H. Witt, N.G. GottardoPediatric brain tumors: innovative genomic information is transforming the diagnostic and clinical landscape $\mathrm{J}$ Clin Oncol, 33 (27) (2015 Sep 20), pp. 2986-2998, 10.1200/JCO.2014.59.9217 Epub 2015 Aug 24. Review

14. D.W. Ellison, M. Kocak, D. Figarella-Branger, G. Felice, G. Catherine, T. Pietsch, et al.Histopathological grading of pediatric ependymoma: reproducibility and clinical relevance in European trial cohorts J Negat Results Biomed, 10 (2011 May 31), p. 7, 10.1186/1477-5751-10-7

15. R. Capocaccia, G. Gatta, P. Roazzi, E. Carrani, M. Santaquilani, R. De Angelis, et al.The EUROCARE-3 database: methodology of data collection, standardisation, quality control and statistical analysis Ann Oncol, 14 (Suppl. 5) (2003), pp. 14-27

16. O. Visser, E. Ardanaz, L. Botta, M. Sant, A. Tavilla, P. Minicozzi, et al.Survival of adults with primary malignant brain tumours in Europe; Results of the EUROCARE-5 study Eur J Cancer (2015 Sep 5)

17. D. Meyroneta, K. Silva, D. Figarella-Branger, C. Godfraind, M.B. Delisle, C.A. Maurage, et al.Réseau de relecture des tumeurs pédiatriques du système nerveux central par le Groupe d'étude de neuropathologie oncologique pédiatrique (GENOP) Ann Pathol, 34 (1) (2014), pp. 74-86

18. European Commission [Internet] Horizon 2020: Spreading excellence and widening participation [cited 2016 November 10] Available from:

https://ec.europa.eu/programmes/horizon2020/en/h2020-section/spreading-excellence-andwidening-participation.

19. Official Journal of the European Union [Internet] Directive 2011/24/EU of the European Parliament and of the Council on the applications of patients' rights in cross-border health care [updated 2011 March 9; cited 2016 November 10]. Available from: http://eurlex.europa.eu/LexUriServ/LexUriServ.do?uri=OJ:L:2011:088:0045:0065:en:PDF. 